\title{
A New Nomenclature of Xenopus laevis Chromosomes Based on the Phylogenetic Relationship to Silurana/Xenopus tropicalis
}

\author{
Yoichi Matsuda $^{a}$ Yoshinobu Uno $^{a}$ Mariko Kondo $^{b}$ Michael J. Gilchrist ${ }^{d}$ \\ Aaron M. Zorn ${ }^{\mathrm{e}}$ f Daniel S. Rokhsarg, h Michael Schmid ${ }^{\mathrm{i}}$ Masanori Taira ${ }^{\mathrm{c}}$ \\ a Laboratory of Animal Genetics, Department of Applied Molecular Biosciences, Graduate School of Bioagricultural \\ Sciences, Nagoya University, Nagoya, ${ }^{b}$ Misaki Marine Biological Station, Graduate School of Science, and Center \\ for Marine Biology, The University of Tokyo, Miura, and ' Department of Biological Sciences, Graduate School of \\ Science, The University of Tokyo, Tokyo, Japan; d Division of Systems Biology, MRC National Institute for Medical \\ Research, London, UK; ' Division of Developmental Biology, Perinatal Institute, Cincinnati Children's Hospital, \\ and $\mathrm{f}$ Department of Pediatrics, College of Medicine, University of Cincinnati, Cincinnati, Ohio, 9 Department of \\ Energy Joint Genome Institute, Walnut Creek, Calif., and h Department of Molecular and Cell Biology, University of \\ California, Berkeley, Calif., USA; 'Department of Human Genetics, University of Würzburg, Würzburg, Germany
}

\section{Key Words}

BrdU replication banding pattern $\cdot$ Homoeologous chromosomes $\cdot$ Nomenclature $\cdot$ Xenopus laevis $\cdot$ Xenopus tropicalis

\begin{abstract}
Xenopus laevis (XLA) is an allotetraploid species which appears to have undergone whole-genome duplication after the interspecific hybridization of 2 diploid species closely related to Silurana/Xenopus tropicalis (XTR). Previous cDNA fluorescence in situ hybridization (FISH) experiments have identified 9 sets of homoeologous chromosomes in X. laevis, in which 8 sets correspond to chromosomes $1-8$ of $X$. tropicalis (XTR1-XTR8), and the last set corresponds to a fusion of XTR9 and XTR10. In addition, recent $X$. laevis genome sequencing and BAC-FISH experiments support this physiological relationship and show no gross chromosome translocation in the $X$. laevis karyotype. Therefore, for the benefit of both comparative cytogenetics and genome research, we here propose a new chromosome nomenclature for $X$. laevis based on the phylogenetic relationship and chromosome
\end{abstract}

\section{KARGER 125}

() 2015 S. Karger AG, Basel

$1424-8581 / 15 / 1454-0187 \$ 39.50 / 0$

E-Mail karger@karger.com

www.karger.com/cgr length, i.e. XLA1L, XLA1S, XLA2L, XLA2S, and so on, in which the numbering of XLA chromosomes corresponds to that in $X$. tropicalis and the postfixes ' $\mathrm{L}$ ' and ' $\mathrm{S}$ ' stand for 'long' and 'short' chromosomes in the homoeologous pairs, which can be distinguished cytologically by their relative size. The last chromosome set is named XLA9L and XLA9S, in which XLA9 corresponds to both XTR9 and XTR10, and hence, to emphasize the phylogenetic relationship to $X$. tropicalis, XLA9_10L and XLA9_10S are also used as synonyms.

(c) 2015 S. Karger AG, Basel

Xenopus laevis (XLA) is allotetraploid and has 36 chromosomes $(4 \mathrm{x}=2 \mathrm{n}=36)$, consisting of 2 sets of 18 chromosomes, which are cytologically distinguished [Wickbom, 1945; Weiler and Ohno, 1962; Morescalchi, 1963; Tymowska and Kobel, 1972]. Its relative X. tropicalis

\section{Yoichi Matsuda}

Laboratory of Animal Genetics, Department of Applied Molecular Biosciences Graduate School of Bioagricultural Sciences, Nagoya University

Furo-cho, Chikusa-ku, Nagoya, Aichi 464-8601 (Japan)

E-Mail yoimatsu@agr.nagoya-u.ac.jp

Masanori Taira

Department of Biological Sciences, Graduate School of Science

The University of Tokyo

7-3-1 Hongo, Bunkyo-ku, Tokyo 113-0033 (Japan)

E-Mail m_taira@bs.s.u-tokyo.ac.jp 
(XTR) is diploid and has 20 chromosomes $(2 \mathrm{x}=2 \mathrm{n}=20)$. The conventionally stained karyotypes of the genus Xenopus, including those of $X$. laevis and various other subspecies, were presented and reviewed in detail by Tymowska [1977, 1991]. A high-resolution replication-banding karyotype of X. laevis was first published by Schmid and Steinlein [1991]. In that study, the chromosomes were arranged according to the karyotype system established by Tymowska and Kobel [1972] and Tymowska [1991], and the identification of homoeologous chromosomes (quartets; each quartet consists of a homoeologous set of 2 homologous chromosomes) was attempted on the basis of similarities in morphology and banding patterns.

In recent years, detailed analyses of the homoeologous chromosomes of X. laevis have been conducted at the molecular level. To investigate the process of genomic and chromosomal reorganization in X. laevis after allotetraploidization, chromosome painting of $X$. laevis was carried out using DNA probes made from microdissected $X$. tropicalis chromosomes [Krylov et al., 2010]. Subsequently, comparative cytogenetic mapping of functional genes between $X$. tropicalis and X. laevis was performed by chromosome fluorescence in situ hybridization (FISH) using 60 cDNA clones derived from $X$. laevis, which covered all $X$. tropicalis chromosomes [Uno et al., 2013]. By these molecular cytogenetic analyses of chromosome homoeologies between the 2 species, all 9 pairs of homoeologous chromosomes were precisely identified at the molecular level. In parallel, the $X$. laevis genome project was progressed by the US-Japan X. laevis Genome Project Consortium, further confirming the data of Uno et al. [2013] on the homoeologous X. laevis chromosomes (quartets). Therefore, this is the time to propose a new nomenclature of $X$. laevis chromosomes based on both molecular and cytogenetic data, which easily allows identifying the phylogenetic relationship of the homoeologous chromosome pairs of $X$. laevis and X. tropicalis. In this new nomenclature, the numbering of $X$. laevis chromosomes corresponds to that in X. tropicalis, and the postfixes ' $L$ ' and 'S' stand for 'long' and 'short' chromosomes in the homoeologous pairs of X. laevis. We also propose a standard idiogram of BrdU-banded X. laevis chromosomes to indicate the position of the genes mapped by in situ hybridization.

\section{Materials and Methods}

Late-replication banding with 5-bromodeoxyuridine (BrdU) and Hoechst 33258 staining of $X$. laevis chromosomes was performed according to Uno et al. [2008, 2013]. For this chromosome banding, fibroblast cells derived from heart or kidney tissues were

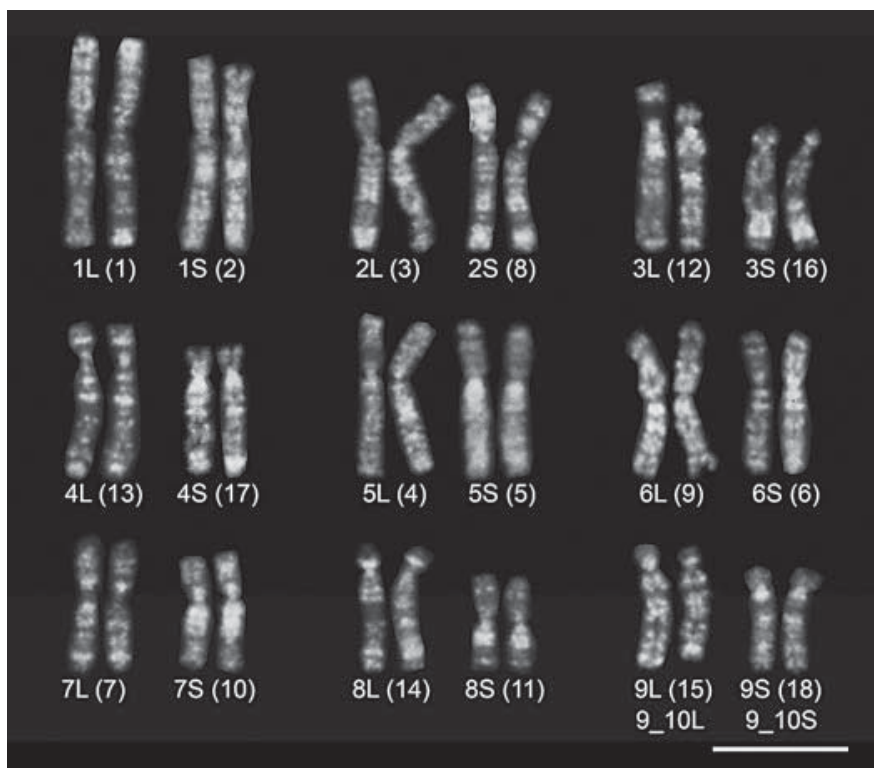

Fig. 1. Hoechst 33258-stained late-replication-banded karyotype of $X$. laevis chromosomes from cultured fibroblasts. The lightly stained bands correspond to G-positive bands produced by GTGbanding (G-bands by trypsin using Giemsa) in mammalian and avian chromosomes. The homoeologous chromosomes are arranged in quartets and numbered according to the new nomenclature system. The numbers in parentheses correspond to the chromosome numbers used by Tymowska and Kobel [1972], Tymowska [1991], Schmid and Steinlein [1991], and Uno et al. [2013]. Scale bar $=10 \mu \mathrm{m}$. Image modified from Uno et al. [2013].

cultured at $26^{\circ} \mathrm{C}$ and treated in the late S-phase with $25 \mu \mathrm{g} / \mathrm{ml}$ BrdU for $6 \mathrm{~h}$ before harvesting. Chromosome preparations were made following a standard air-drying method. After staining with Hoechst $33258(1 \mu \mathrm{g} / \mathrm{ml})$ for $5 \mathrm{~min}$, the slides were heated to $65^{\circ} \mathrm{C}$ for $3 \mathrm{~min}$ on a hotplate and then exposed to UV light for an additional 5-6 $\mathrm{min}$ at $65^{\circ} \mathrm{C}$.

Chromosome lengths were measured using 5 metaphase chromosome spreads from 3 females, which were prepared as described above. Each chromosome or arm length was normalized by the total chromosome length of the respective metaphase.

\section{Results and Discussion}

Recent X. laevis genome analyses and chromosome FISH experiments using about $800 \mathrm{BAC}$ clones confirmed all 9 quartets of chromosomes, which were previously identified by cDNA FISH [Uno et al., 2013], and also demonstrated that there are no gross interchromosomal rearrangements [unpublished data of the US-Japan $X$. laevis Genome Project Consortium co-led by D.S.R. and M.T.]. Figure 1 shows the BrdU/Hoechst late-replication 
Table 1. Numbering system of $X$. tropicalis and X. laevis chromosomes based on their homoeologies, chromosome lengths, centromere indices, and average length ratios of short to long chromosomes in each of the 9 chromosome quartets of $X$. laevis

\begin{tabular}{|c|c|c|c|c|c|}
\hline \multicolumn{3}{|c|}{ Chromosome numbering system } & \multirow{2}{*}{$\begin{array}{l}\text { Chromosome length } \\
(p+q \text { arm }), \%^{c}\end{array}$} & \multirow{2}{*}{$\begin{array}{l}\text { Centromere } \\
\text { index, } \% \mathrm{~d}\end{array}$} & \multirow{2}{*}{$\begin{array}{l}\text { S/L length } \\
\text { ratio } \pm \mathrm{SD}^{\mathrm{e}}\end{array}$} \\
\hline X. tropicalis ${ }^{\mathrm{a}}$ & $X$. laevis new & X. laevis old ${ }^{\mathrm{b}}$ & & & \\
\hline \multirow[t]{2}{*}{ XTR1 } & XLA1L & 1 & $7.64(3.38+4.26)$ & 44.2 & \multirow[t]{2}{*}{$0.91 \pm 0.01$} \\
\hline & XLA1S & 2 & $6.96(2.80+4.16)$ & 40.2 & \\
\hline \multirow[t]{2}{*}{ XTR2 } & XLA2L & 3 & $6.68(2.61+4.07)$ & 39.1 & \multirow[t]{2}{*}{$0.94 \pm 0.03$} \\
\hline & XLA2S & 8 & $6.28(2.15+4.13)$ & 34.2 & \\
\hline \multirow[t]{2}{*}{ XTR3 } & XLA3L & 12 & $6.69(1.70+4.99)$ & 25.4 & \multirow[t]{2}{*}{$0.75 \pm 0.01$} \\
\hline & XLA3S & 16 & $5.05(1.16+3.89)$ & 23.0 & \\
\hline \multirow[t]{2}{*}{ XTR4 } & XLA4L & 13 & $5.60(1.36+4.24)$ & 24.3 & \multirow[t]{2}{*}{$0.86 \pm 0.03$} \\
\hline & XLA4S & 17 & $4.79(1.20+3.59)$ & 25.1 & \\
\hline \multirow[t]{2}{*}{ XTR5 } & XLA5L & 4 & $5.92(2.28+3.64)$ & 38.5 & \multirow[t]{2}{*}{$0.92 \pm 0.04$} \\
\hline & XLA5S & 5 & $5.46(1.89+3.57)$ & 34.6 & \\
\hline \multirow[t]{2}{*}{ XTR6 } & XLA6L & 9 & $5.91(2.74+3.17)$ & 46.4 & \multirow[t]{2}{*}{$0.90 \pm 0.03$} \\
\hline & XLA6S & 6 & $5.34(2.12+3.22)$ & 39.7 & \\
\hline \multirow[t]{2}{*}{ XTR7 } & XLA7L & 7 & $4.88(2.08+2.80)$ & 42.6 & \multirow[t]{2}{*}{$0.90 \pm 0.04$} \\
\hline & XLA7S & 10 & $4.37(1.92+2.45)$ & 43.9 & \\
\hline \multirow[t]{2}{*}{ XTR8 } & XLA8L & 14 & $5.17(1.28+3.89)$ & 24.8 & \multirow[t]{2}{*}{$0.75 \pm 0.05$} \\
\hline & XLA8S & 11 & $3.87(1.79+2.08)$ & 46.3 & \\
\hline \multirow[t]{2}{*}{ XTR9 + XTR10 } & XLA9L (9_10L) & 15 & $5.00(1.10+3.90)$ & 22.0 & \multirow[t]{2}{*}{$0.88 \pm 0.03$} \\
\hline & XLA9S (9_10S) & 18 & $4.41(1.11+3.30)$ & 25.2 & \\
\hline
\end{tabular}

${ }^{a}$ Numbering used by Khohka et al. [2009]. ${ }^{b}$ Numbering used by Tymowska and Kobel [1972], Tymowska [1991], Schmid and Steinlein [1991], and Uno et al. [2013]. ' Ratio of individual chromosome length to the total length of all chromosomes. ${ }^{\mathrm{d}}$ Ratio of the short arm length to the total chromosome length. ${ }^{\mathrm{e}}$ Average ratio calculated for each of the 4 chromosomes in the quartets.

banding patterns that are unique to each $X$. laevis chromosome and have been used for identification of chromosomes and physical assignment of loci on the chromosomes. Based on this chromosome identification, we measured the lengths of short and long chromosome arms and compared the total lengths of the 2 homoeologous chromosome sets in each quartet. Table 1 shows that the length ratio of shorter versus longer chromosome of each homoeologous pair ranges from $0.75 \pm 0.01$ to 0.94 \pm 0.03 . Therefore, in each of the quartets, homoeologous chromosome sets can be distinguished based on their overall lengths in addition to their specific banding patterns, with one chromosome pair being long (L) and the other being short (S) (fig. 1; table 1).

Based on the highly conserved synteny of $X$. laevis and $X$. tropicalis chromosomes [Uno et al., 2013], their unique chromosome banding patterns and length differences be-

New Nomenclature of Xenopus laevis Chromosomes tween homoeologous chromosomes, we propose a new nomenclature of $X$. laevis chromosomes that reflects the phylogenetic relationship with $X$. tropicalis chromosomes as shown in figures 1 and 2 . In this nomenclature system, we used $X$. tropicalis as the reference, because it is the extant diploid species closest to X. laevis, in which its 10 pairs of chromosomes are traditionally arranged by decreasing lengths and numbered consecutively from 1 through 10 (XTR1-XTR10) [Khokha et al., 2009]. We designated X. laevis chromosomes as XLA1, XLA2, and so on for each homoeologous pair, where the XLA1 pair, for example, phylogenetically corresponds to XTR1. The designations $\mathrm{L}$ or $\mathrm{S}$ were further added to the chromosome numbers as postfixes (XLA1L, XLA1S, XLA2L, $\mathrm{XLA2S}$, and so on) to distinguish the long and short chromosomes of a homoeologous set in each quartet (fig. 2; table 1). 


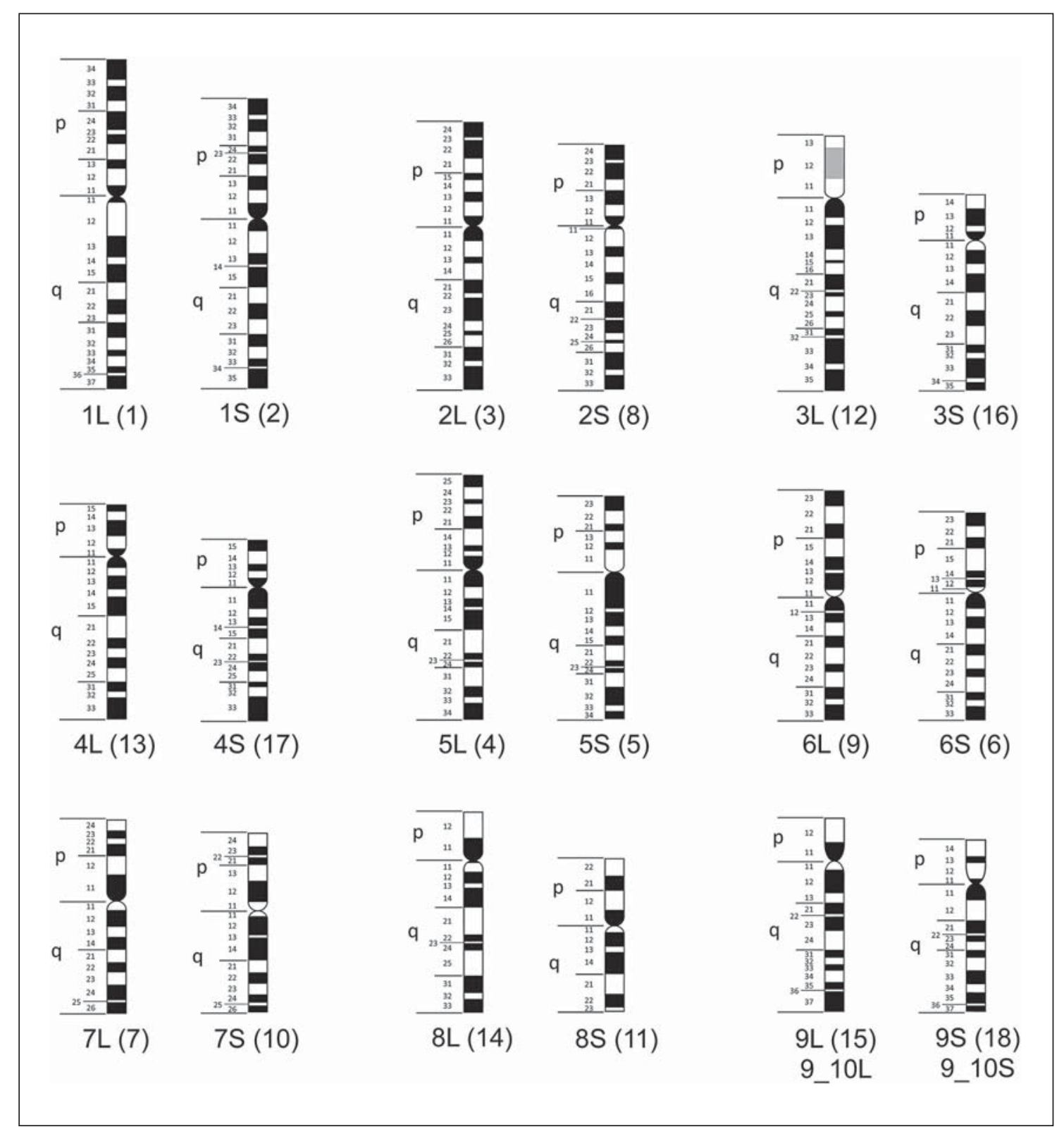

Fig. 2. Idiogram of the late-replication banding patterns of X. laevis chromosomes stained with Hoechst 33258. The chromosomes are numbered according to the new nomenclature system. The numbers in parentheses correspond to the chromosome numbers used by Uno et al. [2013]. The grey band (p12) in chromosome $3 \mathrm{~L}$ is the nucleolus organizer region.

XTR1 to XTR8 phylogenetically correspond to pairs XLA1 to XLA8, respectively, with the same consecutive numbering. However, the last $2 X$. tropicalis chromosomes (XTR9 and XTR10) are homoeologous to the single 9 th chromosome pair of $X$. laevis. It is likely that these chromosomes derived from a fusion event of 2 chromosomes that correspond to XTR9 and XTR10 in the ances- tral lineage of X. laevis [unpublished data of the US-Japan $X$. laevis Genome Project Consortium]. Although each of these chromosomes corresponds to XTR9 and XTR10, we designated them as XLA9L and XLA9S. Alternatively, the 9th $X$. laevis chromosomes can also be referred to as XLA9_10L and XLA9_10S as synonyms of XLA9L and XLA9S, respectively, because this designation is easily un- 
derstandable and takes into account the chromosome rearrangement that occurred in karyotype evolution (tandem fusion of 2 chromosomes followed by centromere repositioning). Cytogeneticists might prefer XLA9L and XLA9S to prevent the confusion due to yet unidentified and undetectable chromosomal rearrangements that may have occurred in the ancestral genomes. However, the designation XLA9_10L and XLA9_10S is convenient for comparative genomics of $X$. tropicalis and $X$. laevis, which are used extensively as model organisms for biomedical research where easy direct genome/chromosome comparisons between the 2 species are extremely valuable. However, this system should be applied only to Xenopus and its closely related species but not to any other farrelated species beyond Xenopodinae whose chromosomes might have been rearranged extensively [Morescalchi, 1968; Tymowska, 1991]. We note that this system is not suited to cope with complex chromosome reshufflings, especially reciprocal translocations, in karyotypes of even closely related species.

The designation of chromosomes in an allopolyploid karyotype should reflect the ancestral diploid genomes prior to hybridization, but need not indicate every chromosome rearrangement that may have occurred before and after the hybridization event. However, in the present case of $X$. laevis, there is no evidence of complex karyotype repatterning because the synteny of each chromosome is highly conserved in X. tropicalis and X. laevis [Uno et al., 2013; the $X$. laevis genome sequence ver. 7.1 at http://gbrowse.xenbase.org/fgb2/gbrowse/xl7_1/; unpublished BAC-FISH data of the US-Japan X. laevis Genome Project Consortium].

The $X$. laevis chromosome nomenclature system proposed here has been approved on September 9, 2014 by the Xenopus Gene Nomenclature Committee chaired by A.M.Z. (http://www.xenbase.org/gene/static/ geneNomenclature.jsp). The complete Xenopus chromosome nomenclature guidelines can be found on Xenbase (http://www.xenbase.org/), the Xenopus model organism database [Karpinka et al., 2014].

\section{Acknowledgements}

We thank all members of the Xenopus Gene Nomenclature Committee for participating in the discussions on the X. laevis chromosome nomenclature system. We also thank all members of the US-Japan X. laevis Genome Project Consortium for sharing the BAC-FISH data before publication. This work was supported in part by a Grant-in-Aid for Scientific Research on Innovative Areas (No. 23113004) from the Ministry of Education, Culture, Sports, Science and Technology of Japan to Y.M.

\section{References}

Karpinka JB, Fortriede JD, Burns KA, James-Zorn Morescalchi A: I cromosomi di alcuni Pipidae C, Ponferrada VG, et al: Xenbase, the Xenopus model organism database; new virtualized system, data types and genomes. Nucleic Acids Res 43(database issue):D756-D763 (2014).

Khokha MK, Krylov V, Reilly MJ, Gall JG, Bhattacharya D, et al: Rapid gynogenetic mapping of Xenopus tropicalis mutations to chromosomes. Dev Dyn 238:1398-1406 (2009).

Krylov V, Kubickova S, Rubes J, Macha J, Tlapakova T, et al: Preparation of Xenopus tropicalis whole chromosome painting probes using laser microdissection and reconstruction of $X$. laevis tetraploid karyotype by Zoo-FISH. Chromosome Res 18:431-439 (2010).

Morescalchi A: Conferma della presenza di eterocromosomi in Xenopus laevis Daudin. Rend Acc Sci (Napoli), Ser 4 30:310-314 (1963). (Amphibia Salientia). Experientia 24:81-82 (1968).

Schmid M, Steinlein C: Chromosome banding in Amphibia. XVI. High-resolution replication banding patterns in Xenopus laevis. Chromosoma 101:123-132 (1991).

Tymowska J: A comparative study of the karyotypes of eight Xenopus species and subspecies possessing a 36-chromosome complement. Cytogenet Cell Genet 18:165-181 (1977).

Tymowska J: Polyploidy and cytogenetic variation in frogs of the genus Xenopus; in Green DS, Sessions SK (eds): Amphibian Cytogenetics and Evolution, pp 259-297 (Academic Press, San Diego 1991).

Tymowska J, Kobel HR: Karyotype analysis of Xenopus muelleri (Peters) and Xenopus laevis (Daudin), Pipidae. Cytogenetics 11:270-278 (1972).
Uno Y, Nishida C, Yoshimoto S, Ito M, Oshima $\mathrm{Y}$, et al: Diversity in the origins of sex chromosomes in anurans inferred from comparative mapping of sexual differentiation genes for three species of the Raninae and Xenopodinae. Chromosome Res 16:999-1011 (2008).

-Uno Y, Nishida C, Takagi C, Ueno N, Matsuda Y: Homoeologous chromosomes of Xenopus laevis are highly conserved after wholegenome duplication. Heredity 111:430-436 (2013).

Weiler C, Ohno S: Cytological confirmation of female heterogamety in the African water frog (Xenopus laevis). Cytogenetics 1:217-223 (1962).

Wickbom T: Cytological studies on Dipnoi, Urodela, Anura, and Emys. Hereditas 31:241-346 (1945). 